\title{
Necessity in Kant: Subjective and Objective
}

\author{
DAVID T. LARSON \\ University of Kansas
}

Kant suggests that his contribution to philosophy is analogous to the contribution of Copernicus to astronomy--each involves the revolutionary reorientation of a discipline. Kant describes his revolution as the move from taking objects to be determinative of knowledge to taking knowledge to be determinative of objects. This revolution should be seen in its proper context; it is Kant's response to such philosophers as Descartes, Leibniz, Newton, and Hume. Descartes initiated a new approach to philosophy, a new method, where the central concern of this approach was epistemological. Descartes posed the questions about the possibility and certainty of knowledge which philosophers through Kant regarded as fundanental. With respect to philosophy done within this epistemological context, Kant's contribution is revolutionary in showing the concept of an object to be dependent on the conditions for the knowledge of objects. Kant is, in a stronger way, also revolutionary with respect to philosophy which preceded the epistemological context. Kant makes the most of the new method he shares with Descartes; he does not only, with Descartes, take epistemology to be fundamental to philosophy, but he also recognizes that the notion of an object must itself be an epistemological notion.

I wish first to provide something of a characterization of the epistemological method in philosophy. To the extent that the characterization is successful, it will support the claim that there is such a common method. Whether or not the method is fully shared with anyone else, however, I wish to explicate its role in Kant's critical philosophy.

Philosophy has long laid special claim to being rigorous. It has claimed a role comparable only to mathematics in developing consistent, if not certain, systems of thought. In reflecting upon itself, philosophy must reflect upon logic or logical reasoning, and in doing this it must provide an interpretation of necessary connection. The interpretation chosen affects to a very great degree the perception which philosophy has of itself, its task, and its method. There appear to be three sorts of interpretations which can be 
chosen. Aristotle, for example, finds the primary sort of necessity to be causal necessity between things in the world. Syllogistic necessity is interpreted as causal necessity. Descartes assumes a psychological interpretation of necessity. Reasoning should procede only by way of such connections as cannot be thought otherwise if one's conclusions are to be logically certain. If two ideas can be thought other than as connected, that is, if their connection can be doubted, then necessary reasoning will not depend on the connection. Wittgenstein displays a third interpretation when he says that all necessity is linguistic.

Kant assumes a psychological interpretation of necessity. The interpretation is psychological in a broad sense, psychological as opposed to physical or linguistic. Something is necessary if it cannot be thought otherwise. This is subjective necessity. It has to do with what can or cannot be thought, not with what can or cannot be. If necessity is primarily psychological, then physical and linguistic necessity, if there be such, must be understood in terms of psychological necessity. If necessity is primarily subjective, then objective necessity, if there be such, must be understood in terms of subjective necessity. It is in a methodological sense that psychological necessity is prior to any other necessity. To say that psychological necessity is primary is to claim that it must be first in the order of understanding, or at least, to suggest that it be taken as such. It is not in itself a claim that psychological necessity has other than subjective validity, although it must arguably lead to such a claim. The choice of a psychological interpretation is a beginning point of philosophy, a fundamental choice of method, not the sort of thing which can be argued for, although the choice is presumably dependent on the prospects for profitable employment of the method. With the choice of the psychological interpretation comes the epistemological problem as fundamental. This problem is the problem of the establishment of the objective necessity of subjectively necessary conclusions. Objective necessity must either be argued for from subjective necessity, or abandoned. Descartes offered a theistic version of such an argument; Hume abandoned objective necessity. The epistemological problem must, in the Cartesian context, be dealt with before any other, because the way in which reason applies cannot be understood until objective necessity is either established or abandoned.

The psychological interpretation takes necessity to be first understandable as necessary connection between mental entities, or representations. Representations which cannot be separated in thought are necessarily connected. The thought of the one makes the thought of the other in some sense unavoidable. Necessary connection might not be immediately evident. It might well 
be quite difficult to determine whether or not two representations are necessarily connected, and some sort of close introspective examination which methodically attempts the separation might well have to be undertaken. Necessary connection, however, does not depend on its recognition as such. Someone might well be mistaken in his judgements of necessary connection even though this judgement requires only introspective examination. Representations, it is assumed, are perfectly definite entities, and their inter-relations do not depend on being recognized. Another important assumption is that error can, with care, be avoided. The mind is open to its own inspection, and if it will only procede with all possible caution it will avoid introspective error.

Kant calls representations "modifications of the mind." I Intuitions and concepts are both representations. ${ }^{2}$ Intuitions are representations of objects; concepts are representations of representations. A single concept subsumes many representations. Judgements subsume representations under concepts, and are the sorts of things which can be true or false, analytic or synthetic, a priori or a posteriori, necessary or contingent.

The notion of a necessary judgement is Kant's fundamental notion of necessity. It is this notion which is Kant's understanding or interpretation of logical necessity. Logical necessity is for Kant something psychological, or conceptual; it is neither substantial nor linguistic. Because the notion of a necessary judgement is fundamental, it is not very descriptive of necessary judgements to say that they are judgements which are logically necessary. Logical necessity must itself be understood either as substantial, psychological, or linguistic necessity; and Kant has chosen to understand it as psychological necessity. To say, then, that a necessary judgement is a judgement which is logically necessary is to seek to explain one notion by another which is dependent on $1 t$.

A necessary judgement is a judgement of a matter which cannot be thought otherwise. Said this simply. however, it sounds as though judgements contrary to necessary judgements are never made. Such judgements-that is. logical errors--are of course made regularly. There is then a sense in which the matter of a necessary judgement can be thought otherwise. In another sense, however, it cannot be thought otherwise. This sense depends on the identification of a set of ideal conditions of judgement under which no judgements contrary to necessary judgements can be made. Logical errors, that is, could not be made under such ideal conditions. Descartes is suggestive of the sorts of conditions that would constitute ideal conditions. The conditions would surely include Descartes' criteria of certainty, namely clarity and distinctness of concep- 
tion. Judgements concerning concepts the conceptions of which are recognizably unclear can easily be thought otherwise, that is, they are not necessary. Descartes seems to suggest that, if only we can get the appropriate concepts perfectly clear, we will not err in our judgements. Judgements made under ideal conditions are judgements made on the basis of concepts alone, for it is only with concepts that the requisite clarity can be hoped for.

All necessary judgements are a priori, and all a priori judgements are necessary. A priori judgements are such judgements as Kant caliss pure, or free from any empirical element. These judgements are not made on the basis of any particular experience, nor do they depend on any other judgements so made. ' A judgement which is not a priori is called a posteriori. Necessary judgements are judgements which cannot be thought otherwise, but any judgement made on the basis of experience can be thought otherwise. If on the basis of experience one representation is subsumed under another, the judgement could be otherwise because the empirical element can be thought otherwise. It is just in case a judgement is not based on experience that there is nothing about the judgement which can be thought otherwise and the judgement is necessary.

$A$ judgement is analytic if it is explicative of a concept; synthetic, if it connects a representation with another which is not a part of it. Concepts are rules for the introduction of unity into a manifold." They are rules according to which different representations are treated as identical in some respect. Concepts synthesize diversity into unity. Concepts may be quite complex; the sum total of a variety of distinguishable rules functioning together. As a more complex rule incorporates simpler rules, so a complex concept incorporates, or includes, simpler concepts. The simpler are internal to the complex. A complex concept is dependent on those internal to it in a way in which they are not dependent on it. The use of the more simple does not involve the use of the more complex as its use does theirs. Saying that one concept is internal to another means only that the use of the latter involves the use of the former; it does not mean that the former can only be used in the latter. A concept can be confusedly thought; that is, the concepts internal to it might not be recognized as such." One concept might not be recognized to depend on other concepts when it does in fact depend on them, just as it might not be recognized that the following of one rule involves the following of certain others. An analytic judgement is a clarification of a concept. It is the recognition that a certain predicate concept is internal to a subject concept. The subject concept incorporates, or depends on, the predicate concept. Because an analytic judgement only recognizes this 
dependence, it adds nothing to the subject concept. That is, it does not further qualify or determine the subject. An analytic judgement does not mark off a limited use of the subject concept--a use restricted by the predicate concept--but recognizes an element of the subject concept which is common to its every possible use. An analytic judgement is a necessary judgement because the (clear) thought of the subject involves the thought of the predicate. An analytic judgement cannot be thought otherwise.

Judgements are either analytic or synthetic. Synthetic judgements, instead of discovering a second concept in a first, bring together two representations external to one-another. A predicate is not found in, but added to, a subject. A synthetic judgement makes a further qualification of the subject, which is thought to be, not just itself (as it would be in an analytic, or 1dentical, judgement), but something else as well. The use of the concept as so judged is a use restricted by the predicate concept.

Every judgement is either a priori or a posteriori and either analytic or sythetic. No judgement. can be analytic a posteriori. Analytic judgements depend only on the clear recognition of concepts, but a posteriorl judgements are based on experience. All analytic judgements are a priori. All a posteriori judgements are therefore synthetic. Both a priori analytic judgements and a posteriori synthetic judgements are possible, and the bases for making them are easily seen. Analytic judgements are made when the understanding gives consideration to its own concepts. The basis of an analytic judgement is the clear thought of a concept, for an analytic judgement is a judgement only of what is internal to a concept. Synthetic judgements connect a subject with an external predicate, a concept not thought in the thought of the subject. Synthetic judgements cannot therefore be based simply on the thought, however clear, of the subject. The understanding must discover the connection somewhere else, in something other than the concepts judged. Experience can provide a basis for synthetic judgements, thus connecting concepts which are not internally connected, but judgements based on experience are a posteriori synthetic.

Analytic judgements are paradigms of necessary judgements. The thought of the subject cannot be separated from the thought of the predicate. Analytic judgements do not go beyond concepts; they are not judgements about experience, but judgements about the possibilities of thought. An analytic judgement simply points out that one concept is internal to another and that it is therefore unavoidable that any use of the latter concept involve the use of the former. The ground of an analytic judgement is the identity which a concept has with itself, and it is because no concept 
can be thought as other than itself that an analytic judgement is a necessary judgement. The ground of an analytic judgement is a connection internal to a concept.

Concepts external to one-another can also be connected. When the connection is a matter of experience--a matter, that is, of conjunctions of intuitions--the synthetic judgement of the connection is never necessary, because the various intuitions can always be imagined separate. A posteriori synthetic judgements are not necessary judgements because, as Hume shows, any two impressions, though conjoined in experience, can be separated in thought. Are there, however, any synthetic judgements which are necessary? Are there any connections which cannot be thought otherwise between concepts external to one-another? since only a priorl judgements can be necessary, the question concêrns the possibility of a priori synthetic judgements.

The basis of an a priori synthetic judgement could not be the identity of a concept, else the judgement would be analytic; nor the conjunction of intuitions, else it would be a posteriori and not necessary. A priori synthetic judgements can be shown possible onl $\vec{y}$ by finding some other ground on the basis of which judgements can be made. objects are the sorts of things of which we are given appearances through intuition and to which concepts apply. objects, however, cannot provide the ground of any judgements. The understanding does not have direct access to objects, but only to appearances of objects, and any judgement based on appearances is contingent. Connections of appearances can always be thought different.

Hume found no ground for a priori synthetic judgements, but he did show that objects themselves could not ground judgements. On Hume's account, there is no difference between an idea of an object, and an idea of that object as existent. Hume distinguishes internal from external objects. An object is said to be internal if it 18 directly present to the mind. Impressions and ideas are such objects. An object is said to be external if it can only be presented to the mind through perception. An external object is indirectly present with the mind when the mind has an impression of it. The impression of an external object is an internal object immediately present to the mind. The idea of external existence is the idea of continued and distinct existence." If an existence is not interrupted by, or dependent on, perception, then that existence is external. That a given existence is external cannot, of course, be discovered through perception. Perception cannot discover whether something exists when not perceived. Objects are thought as having external existence only because of the constancy and coherence of their impressions. External existence is 
not discovered, only supposed. Because external existence, or objectivity, is not something that can be discovered, and there is no difference between the idea of an object and the idea of that object as existent, not objects as existent, but only objects as perceived, can ground judgements.

The Humean description of the idea of an (external) object has two main elements. An object has a certain unity (it is one object, not many), and it has continued and distinct existence. The idea of continued and distinct existence is due solely to certain relations between impressions, namely, constancy and coherence.' This idea is not the idea of constancy and coherence, but is a supposition caused by these relations. Unity is also requisite of an object. Before something can be thought to have external existence, it must be thought as a unity. Hume discovers a strong principle of unity operative in human understanding:

We have observed, that whatever objects are different are also distinguishable, and that whatever objects are distinguishable are separable by the thought and imagination. And we may here add, that these propositions are equally true in the inverse, and that whatever objects are separable are also distinguishable, and that whatever objects are distinguishable are also different. 10

Because any two impressions which have been imaginatively associated together can be again separated, this principle declares that each impression is the impression of a different object. This is a principle of subjective necessity. No connections between impressions or ideas are subjectively necessary. Any two impressions or ideas can be imagined separate. The strict Humean notion of an object is a notion of that of which there can be only one impression. Clearly. such a notion cannot provide an account of the way in which all sorts of things which do not meet this strict criterion of unity are nevertheless commonly considered to be objects. Hume therefore proceeds to describe the weakening of the principle of unity by another principle operative in human understanding, namely, the tendency of the imagination to associate closely related impressions. 11 The imagination commonly treats as a unity that which is actually only a collection of impressions. These weak unities are then feigned to represent individual objects. The many things which we commonly consider objects have no necessary unity. An idea of such an object is nothing but the result of pretending that a collection of related impressions has a continued existence distinct from perception.

A synthetic judgement based on an idea of an object depends on the unity of that idea. Only if this unity is a necessary unity can the judgement be necessary. 
Hume finds no such unity. The strict principle of unity finds necessary unity only in indivisible impressions. If, however, no separations are possible, then there can be no synthetic judgements based on that unity. The weaker sort of unity allows for synthetic judgements, but the necessity of the unity disappears. With neither notion of unity are necessary synthetic judgements possible.

Kant finds two grounds for necessary synthetic judgements: pure intuitions and the concept of an object in general. ${ }^{12}$ All appearances cannot but exhibit the forms of intuition. These forms are subjectively necessary in that a condition on something being an appearnce is that it exhibit them. A pure intuition is an intuition which has no empirical element; that is, it is a particular form or rule of intuition. Human beings are, as it happens, so constituted that space and time are the forms of their intuition. Every appearance is thought as being structured by time, the form of inner sense. Those appearances which are given through outer sense are also thought as structured by space. Space and time are neither concepts nor features of things-in-themselves, but ways in which the intuition orders appearances. Appearances, spatially and temporally structured, are the material provided to the understanding. A particular spatial or temporal arrangement without sensible content is a pure intuition. What we might loosely call arithmetical and geometrical objects are pure intuitions. Pure intuitions can be constructed independently of experience. Each construction proceeds according to the rule of some particular arithmetical or geometrical concept.

Pure intuitions are grounds of necessary synthetic judgements. Two geometrical concepts which are external to one-another may nevertheless be judged necessarily connected provided that they are connected in a pure intuition. When geometrical judgements are made, a pure intuition is constructed in accordance with the rule which is the subject concept. Should this intuition be recognized to fall under the predicate concept, it becomes evident that whatever satisfies the subject concept also satisfies the predicate concept, because every appearance is constructed in accordance with the forms of intuition, and it is just that form which is examined when a pure intuition constructed according to one concept is seen to fall under another. The connection of two concepts in a pure intuition is a priori and hence necessary. Synthetic judgements grounded in pure intuitions are necessary just because the exhibition of the forms of intuition is a condition on something being an appearance.

The concept of an object in general is the concept of that something, distinct from representations, of which there may be many representations. The concept of the unity of these many representations is the 
concept of an object. Hume was correct in arguing that the objectivity of an object is precisely that about the object which could never be perceived. Objects as things-in-themselves are wholly unknowable; it is only as objects are represented that they can be known. An object makes necessary a certain unity among representations, that is, the concept of unity among representations is analytic of the concept of an object in general. This unity is only possible as a synthetic unity. The concept of an object is a rule for the synthesis of a manifold. It is only because the unity of an object is a synthetic unity that the unity of experience is possible. The unity of apperception which is analytic of the concept of experience presupposes the synthetic unity of an object. The concepts according to which intuitions are united are necessarily objectively valid because it is precisely the synthetic unity thus created that is thought as an object. The concept of an object in general is necessarily connected with another concept, that of the unity of apperception. These concepts are not analytically, but synthetically connected.

The concept of an object in general, necessarily connected to the concept of the unity of experience as mine, provides a basis for many a priori synthetic judgements. The unity of experience is synthetically provided by the forms of intuition and understanding. These forms are necessarily exhibited by everything insofar as it is thought as an object. That only can be an object to us which exhibits these forms. The forms of intuition are space and time; the forms of understanding are the categories. Objects exhibit to the understanding their unity as objects in their exhibition of the forms of intuition and understanding. This unity is a synthetic unity. Because the unity required by each object turns out to be precisely the synthetic unity given it by the forms of intuition and understanding, the concepts of the forms of intuition and the concepts which are the categories of understanding apply a priori and necessarily to each object. Concepts which are not analytically connected may be found to be necessarily connected by the concept of an object in general. Not because of what is internal to a given concept, but because of what is required by the very possibility of experience, might it be impossible to think that concept other than as connected to some other concept. There is, in other words, another ground for necessary synthetic judgements in addition to the grounds found in pure intuitions. This ground is the synthetic character of an object as the necessary presupposition of the actual unity of apperception.

Kant's fundamental notion of necessity, I have suggested, is a notion of subjective necessity. The necessity of an analytic judgement is the paradigm of 
subjective necessity, because the ground of an analytic judgement is clearly a subjective, or conceptual, ground. The subject concept of an analytic judgement grounds that judgement. The subject concept makes analytic judgements subjectively necessary. Synthetic judgements are different. Neither of the concepts connected in a synthetic judgement are capable of making the judgement subjectively necessary. In discovering that the concept of an object in general makes certain synthetic judgements subjectively necessary, the transcendental deduction not only establishes the possibility of necessary synthetic judgements, but it also establishes a new notion of necessity. This new notion is a notion of objective necessity. Something is objectively necessary if it is required by the possibility of objects. Subjectively necessary judgements grounded on the concept of an object in general are judgements of objective necessity.

The concept of an object in general is the concept of what something must be if it is to be an object. It is the concept of something experienced as a unity. Only that which conforms to the forms of intuition and understanding $c$ an be an object. The concept of an object in general is the concept of a certain unity, a unity which can only be provided by the forms of intuition and understanding. It is necessary that, if something is to be an object, it exhibit this synthetic unity. Each individual object makes necessary a certain unity; that is to say, its very character as an object is dependent on the way in which its concept unifies intuitions according to the categories. It is not the particular unity which is necessary; but the fact that the particular concept introduce a synthetic unity according to the categories. Synthetic judgements grounded on the concept of an object in general are objectively necessary because each particular object cannot but satisfy the concept of an object in general.

The critical character of Kant's philosophy is but another side of the radical re-orientation which is effected in the notion of an object. The re-orientation is encapsulated in the description of an object as "that in the concept of wich a manifold is united." The unity which constitutes an object is a conceptual unity; it is a unity placed upon indeterminate things by the perceiving and understanding subject. Not objects as things-in-themselves, but only objects as appearances have any unity or any determinate character. Hume recognized some of this. He understood that the only sort of unity among things of which persons can have knowledge is such unity as is somehow placed upon those things. Only a subjectively constructed unity is a knowable unity. Hume therefore went to great lengths to describe how it is that the human understanding constructs the unity which is constitutive of an object. 
Hume failed to discover how to establish objective necessity on the basis of subjective necessity. He made a tremendous step in the right direction, however, by avoiding the path taken by Descartes, Leibniz, Newton, and others. These philosophers did not realize that the epistemological project of establishing objective necessity on the basis of subjective necessity could not be consistently carried out by introducing a non-epistemological notion of objectivity. To attempt to found a notion of objectivity on a non-epistemological notion of substance is to proceed in a speculative and dogmatic fashion. Such notions have nothing to do with the discovery of the principles and limits of human understanding. The Kantian re-orientation in the notion of an object is not speculative but critical. Kant does not simply pose one more alternative which can be placed alongside atoms and monads as a conceptually (subjectively) possible ground of the unity constitutive of objects. Kant's alternative is not so much an alternative as 1 t is a solution. It is a conceptual, not a substantial, solution, for it discovers in the concept of an object the ground of objective unity. It discovers a subjective ground of objective necessity. Kant's alternative is a solution in that it discovers that which is necessary for the possibility of unity in experience instead of uncritically suggesting another conceptually un-necessary ground of unity.

\section{NOTES}

Immanuel Kant, Critique of Pure Reason, A98.

${ }^{2}$ Kant, A68, B93.

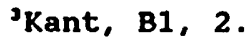

"Kant, A105.

'Kant, A7, B11.

'Kant, B11.

'David Hume, A Treatise of Human Nature, ed. L. A. Selby-Bigge, 2nd ed. revised by $\overline{\mathrm{P}}$. H. Nidditch (Oxford: Oxford University Press, 1978), p. 66.

'Hume, p. 188.

'Hume, pp. 194, 195.

${ }^{\circ}$ Hume, p. 18. 
${ }^{12}$ Hume, p. 263.

${ }^{12}$ Kant, B73. 